University of Nebraska - Lincoln

DigitalCommons@University of Nebraska - Lincoln

4-1-1987

\title{
The breakdown of Hund's rule in systems of fourfold symmetry
}

Gordon A. Gallup

University of Nebraska-Lincoln, ggallup1@unl.edu

Follow this and additional works at: https://digitalcommons.unl.edu/physicsgallup

Part of the Physics Commons

Gallup, Gordon A., "The breakdown of Hund's rule in systems of fourfold symmetry" (1987). Gordon Gallup Publications. 6.

https://digitalcommons.unl.edu/physicsgallup/6

This Article is brought to you for free and open access by the Research Papers in Physics and Astronomy at DigitalCommons@University of Nebraska - Lincoln. It has been accepted for inclusion in Gordon Gallup Publications by an authorized administrator of DigitalCommons@University of Nebraska - Lincoln. 


\title{
The breakdown of Hund's rule in systems of fourfold symmetry
}

\author{
G. A. Gallup \\ Department of Chemistry; University of Nebraska, Lincoln, Nebraska 68588-0304
}

(Received 13 November 1986; accepted 24 December 1986)

\begin{abstract}
The lowest energies of molecular systems with fourfold symmetry can violate Hund's multiplicity rule. We derive relationships between two electron integrals that in favorable cases allow one to predict when this will happen. These relationships result in a fairly simple inequality as a criterion. The systematics of the phenomenon are illustrated with a series of calculations on model systems consisting of hydrogen atoms. These are rings of three, four, five, six, seven, and eight members as well as octahedral and square planar arrangements. These show how the results and simple ideas of molecular orbital theory can be qualitatively modified when various levels of configuration interaction are allowed in the theoretical discription.
\end{abstract}

\section{INTRODUCTION}

Hund's multiplicity rule states that open shell systems in their ground states should have the highest possible multiplicity consistent with the Pauli principle. Consequently, lower multiplicity states are expected to have higher energies. This is one of the most useful generalizations concerning the behavior of $n$-electron systems to come from quantum mechanics. Nevertheless, exceptions, uncovered principally by theoretical calculation, have been observed. Kollmar and Staemmler ${ }^{1}$ give a discussion of the three molecular systems, square planar cyclobutadiene, square planar $\mathrm{H}_{4}$, and $D_{2 d}$ twisted ethylene, and attribute the breakdown of Hund's rule, at least in part, to a phenomenon they call dynamic spin polarization.

In this article we give a systematic discussion of open shell systems of the general configuration $\left(e_{i}\right)^{2}$, where $e_{i}$ represents a pair of orbitals corresponding to a doubly degenerate representation of a molecular point group. We show for any molecular system that has fourfold symmetry, there is at least one $e_{i}$ species for which the $\left(e_{i}\right)^{2}$ configuration is a candidate for a breakdown of Hund's rule. The three molecules above all possess elements of fourfold symmetry $\left(S_{4}\right.$ in $\left.D_{2 h}\right)$ and satisfy our criterion. Conditions other than fourfold symmetry are required, however; certain single excitations in a CI representation of the wave function must make important contributions to lowering the energy. In the analysis we use the version of restricted Hartree-Fock (RHF) theory put forward by Roothaan. ${ }^{2}$ Pople has discussed dynamic spin polarization in terms of the unrestricted Hartree-Fock (UHF) wave functions. ${ }^{3}$

\section{INFLUENCE OF FOURFOLD SYMMETRY}

We consider any two-dimensional representation of one of the point groups $D_{n}, C_{n v}, D_{n h}$, or $D_{n d}$. We do not include $C_{n}$ and $C_{n h}$ in our general formal discussion, since, technically, they have no two-dimensional irreducible representations. Nevertheless, for these latter two groups, the one-dimensional representations that are complex conjugate pairs correspond to energy degeneracy patterns that produce the same results as one gets with the groups we do consider.

A pair of orbitals in a molecule forming a basis for a twodimensional representation, $D\left(R_{p}\right)$, will have the property

$$
\begin{aligned}
& R_{p}\left[e_{i 1} e_{i 2}\right]=\left[e_{i 1} e_{i 2}\right] D\left(R_{p}\right), \\
& \quad=\left[e_{i 1} e_{i 2}\right]\left[\begin{array}{cc}
\alpha_{p} & -\beta_{p} \\
d_{p} \beta_{p} & d_{p} \alpha_{p}
\end{array}\right], \\
& \alpha_{p}=\cos \left(2 \pi n_{p} / k\right), \\
& \beta_{p}=\sin \left(2 \pi n_{p} / k\right), \\
& d_{p}= \pm 1=\operatorname{det}\left[D\left(R_{p}\right)\right],
\end{aligned}
$$

where $n_{p}$ is an integer depending upon the operation $R_{p}$, and $k$ is an integer, $3,4,5, \ldots$, depending on the group and the representation. The value of the determinant is +1 for the proper rotations and -1 for the improper rotations. It should be recalled that even in groups such as $D_{n}$, which consist of only proper three-dimensional rotations, the twodimensional irreducible representation matrices may be either proper or improper.

The states of the configuration $\left(e_{i}\right)^{2}$ for $M_{s}=0$ can be written as linear combinations of the Slater determinantal functions (SDF)

$$
\begin{aligned}
& \phi_{1}=\left|e_{i 1} \bar{e}_{i 1}\right|, \\
& \phi_{2}=\left|e_{i 1} \bar{e}_{i 2}\right|, \\
& \phi_{3}=\left|e_{i 2} \bar{e}_{i 1}\right|, \\
& \phi_{4}=\left|e_{i 2} \bar{e}_{i 2}\right|,
\end{aligned}
$$

where we have suppressed the closed shell portions of the SDFs. Here and later in this article we use $x$ and $\bar{x}$ to represent the spin orbitals with $m_{s}=1 / 2$ and $m_{s}=-1 / 2$ spin functions, respectively.

The transformation (1) induces a transformation among the $\phi_{i}$ 's of the form

$$
\begin{aligned}
& R_{p}\left[\phi_{1} \phi_{2} \phi_{3} \phi_{4}\right] \\
& =\left[\phi_{1} \phi_{2} \phi_{3} \phi_{4}\right] \\
& \quad \times\left[\begin{array}{crrr}
\alpha_{p}^{2} & -\alpha_{p} \beta_{p} & -\alpha_{p} \beta_{p} & \beta_{p}^{2} \\
d_{p} \alpha_{p} \beta_{p} & d_{p} \alpha_{p}^{2} & -d_{p} \beta_{p}^{2} & -d_{p} \alpha_{p} \beta_{p} \\
d_{p} \alpha_{p} \beta_{p} & -d_{p} \beta_{p}^{2} & d_{p} \alpha_{p}^{2} & -d_{p} \alpha_{p} \beta_{p} \\
\beta_{p}^{2} & \alpha_{p} \beta_{p} & \alpha_{p} \beta_{p} & \alpha_{p}^{2}
\end{array}\right] .
\end{aligned}
$$

When we form linear combinations of the $\phi_{i}$ 's that completely reduce the representation based upon them, we get new 
functions that are pure spin states. Three of these new functions are singlet and one is a triplet. Denoting the new functions by ${ }^{1} \psi_{1},{ }^{1} \psi_{2},{ }^{1} \psi_{3}$, and ${ }^{3} \psi$, Eq. (3) becomes

$$
\begin{aligned}
R_{p}\left[{ }^{1} \psi_{1}{ }^{1} \psi_{2}{ }^{1} \psi_{3}{ }^{3} \psi\right] & \\
= & {\left[{ }^{1} \psi_{1}{ }^{1} \psi_{2}{ }^{1} \psi_{3}{ }^{3} \psi\right] } \\
& \times\left[\begin{array}{cccc}
1 & 0 & 0 & 0 \\
0 & \alpha_{p}^{2}-\beta_{p}^{2} & -2 \alpha_{p} \beta_{p} & 0 \\
0 & 2 d_{p} \alpha_{p} \beta_{p} & d_{p}\left(\alpha_{p}^{2}-\beta_{p}^{2}\right) & 0 \\
0 & 0 & 0 & d_{p}
\end{array}\right] .
\end{aligned}
$$

We see that, for most values of $k,{ }^{1} \psi_{2}$ and ${ }^{1} \psi_{3}$ are still a degenerate pair of functions, since they form a two-dimensional representation for the point groups above. Exceptions occur, however. Using Eq. (1) we find that $2 \alpha_{p} \beta_{p}$ $=\sin \left(4 \pi n_{p} / k\right)$, and therefore, if $k=4$, the representation matrix in Eq. (4) is diagonal and, barring accidental degeneracy, three different singlet energies occur.

This lack of spatial degeneracy in the singlet states has, of course, been observed before with systems of fourfold symmetry such as twisted ethylene or square cyclobutadiene or $\mathbf{H}_{4}$. We have demonstrated that it will occur whenever a two-dimensional representation like Eq. (1) has $k=4$.

One might enquire whether similar splittings of a normally degenerate pair of functions occur for other values of $k$. We easily construct examples for such cases. Letting $\left[e_{1} e_{2}\right]$ be the degenerate pair of functions, one sees that

$$
\begin{aligned}
& e_{1}^{(m)}=\left[\left(e_{1}+\mathbf{i} e_{2}\right)^{m}+\left(e_{1}-\mathbf{i} e_{2}\right)^{m}\right] / 2 ; \quad \mathbf{i}=\sqrt{-1}, \\
& e_{2}^{(m)}=-\mathbf{i}\left[\left(e_{1}+\mathbf{i} e_{2}\right)^{m}-\left(e_{1}-\mathbf{i} e_{2}\right)^{m}\right] / 2
\end{aligned}
$$

constitute a pair of real functions that is a basis for a twodimensional representation except when $k=m$.

For the special case $m=3$, Eq. (5) gives

$$
\left[e_{1}^{3}-3 e_{1} e_{2}^{2} 3 e_{1}^{2} e_{2}-e_{2}^{3}\right] \text {, }
$$

and every group with $3 n$-fold symmetry will have at least one two-dimensional representation for which this pair of functions is not also a degenerate pair.

It is now clear, however, that $n=4$ is the only case of interest for fermions. The application of Eq. (5) would require $m$-fold $m>2$ occupancy of orbitals, a circumstance completely prohibited by the Pauli exclusion principle. Therefore, Eq. (5) can apply to fermion states only for $m=2$, and, since spatial degeneracy occurs only for systems with threefold or higher symmetry, $n=4$ is the lowest symmetry for which we can find both degeneracy and its splitting.

\section{A LINEAR RELATION AMONG TWO-ELECTRON INTEGRALS FOR $k \neq 4$}

The symmetry properties of the two-dimensional representations can be used to derive a linear relation among twoelectron integrals over their basis functions. In order to simplify the notation somewhat we denote $\left[e_{1} e_{2}\right]$ as $[u v]$ in this section as well as some later sections. The quadratic functions $\left[\left(u^{2}+v^{2}\right) / 2\left(u^{2}-v^{2}\right) / 2 u v\right]$ form a basis for a completely reduced representation we write (for $k \neq 4$ ) as $A_{1}+E$. Between two sets of these quadratic functions, one set a function of electron 1 and the other set a function of electron 2, we form the cross product representation. This new representation will have a structure we may write as $2 A_{1}+A_{2}+3 E$. (The species designations we use here are specifically those of the group $D_{n}$, but any of the groups we are considering has a corresponding set of symbols.)

The product functions may also be formed into a basis for a completely reduced representation. These are

$$
\begin{aligned}
A_{1}: & (1 / 4)\left[u(1)^{2}+v(1)^{2}\right]\left[u(2)^{2}+v(2)^{2}\right], \\
& (1 / 4)\left[u(1)^{2}-v(1)^{2}\right]\left[u(2)^{2}-v(2)^{2}\right] \\
& +u(1) v(1) u(2) v(2), \\
A_{2}: & (1 / 2)\left\{\left[u(1)^{2}-v(1)^{2}\right] u(2) v(2)\right. \\
& \left.-u(1) v(1)\left[u(2)^{2}-v(2)^{2}\right]\right\}, \\
E: & (1 / 4)\left[u(1)^{2}-v(1)^{2}\right]\left[u(2)^{2}-v(2)^{2}\right] \\
& -u(1) v(1) u(2) v(2), \\
& (1 / 2)\left[u(1)^{2}-v(1)^{2}\right] u(2) v(2) \\
& +u(1) v(1)\left[u(2)^{2}-v(2)^{2}\right], \\
& (1 / 4)\left[u(1)^{2}+v(1)^{2}\right]\left[u(2)^{2}-v(2)^{2}\right], \\
& (1 / 2)\left[u(1)^{2}+v(1)^{2}\right] u(2) v(2), \\
& (1 / 4)\left[u(1)^{2}-v(1)^{2}\right]\left[u(2)^{2}+v(2)^{2}\right], \\
& (1 / 2) u(1) v(1)\left[u(2)^{2}+v(2)^{2}\right] .
\end{aligned}
$$

We now multiply each of these nine functions by $1 / r_{12}$ and integrate over all space for two electrons. Standard group theoretic arguments tell us that only the two $A_{1}$ functions can have a nonzero integral, and we get nine equations for the primitive two electron integrals $J_{u u}, J_{u v}, J_{v v}, K_{u v}$, $L_{u v}(=[u u \mid u v])$, and $L_{v u}$. Not all of these nine equations are independent because integrating the coordinates of both electron 1 and electron 2 over all space makes the expressions equivalent with respect to these two labels. When the redundancies are removed we have the six equations

$$
\begin{aligned}
& S=(1 / 4)\left(J_{u u}+2 J_{u v}+J_{v v}\right), \\
& T=(1 / 4)\left(J_{u u}-2 J_{u v}+J_{v v}\right)+K_{u v}, \\
& 0=(1 / 4)\left(J_{u u}-2 J_{u v}+J_{v v}\right)-K_{u v}, \\
& 0=(1 / 2)\left(L_{u v}-L_{v u}\right), \\
& 0=(1 / 2)\left(L_{u v}+L_{v u}\right), \\
& 0=(1 / 4)\left(J_{u u}-J_{v v}\right),
\end{aligned}
$$

where $S$ and $T$ are two numbers depending on the specific pair of orbitals, [uv].

The last three equations of Eqs. (6) tell us that $L_{u v}$ $=L_{v u}=0$ and $J_{u u}=J_{v v}$. The third of the equations is the linear relation we are seeking and, when rewritten in the light of other equalities, is

$$
0=J_{u u}-J_{u v}-2 K_{u v} \text {. }
$$

We may describe the existence of this relation in another way: The three quantities $J_{u u}, J_{u v}$, and $K_{u v}$ depend upon only the two quantities $S$ and $T$, therefore, they are not completely independent.

An entirely different situation develops when $k=4$. 
The representation based on $\left(u^{2}+v^{2}\right) / 2,\left(u^{2}-v^{2}\right) / 2$, and $u v$ is still in completely reduced form, but the decomposition is now $A_{1}+B_{1}+B_{2}$. There is no degeneracy at this level, and the cross product representation of this with itself now has the decomposition $3 A_{1}+2 A_{2}+2 B_{1}+2 B_{2}$. There are now three independent nonzero integrals, the set of equations corresponding to Eqs. (6) is

$$
\begin{aligned}
& S^{\prime}=(1 / 4)\left(J_{u u}+2 J_{u v}+J_{v v}\right), \\
& T^{\prime}=(1 / 4)\left(J_{u u}-2 J_{u v}+J_{v v}\right)+K_{u v}, \\
& U^{\prime}=(1 / 4)\left(J_{u u}-2 J_{u v}+J_{v v}\right)-K_{u v}, \\
& 0=(1 / 2)\left(L_{u v}-L_{v u}\right), \\
& 0=(1 / 2)\left(L_{u v}+L_{v u}\right), \\
& 0=(1 / 4)\left(J_{u u}-J_{v v}\right),
\end{aligned}
$$

and there is no counterpart to Eq. (7). The normal situation when using group theoretic arguments prevails here: None of these integrals must be nonzero, but those that are not required to be zero we expect to be nonzero. Actually, other properties of the integrals may be used to obtain further information. If $u$ and $v$ are normalizable in the standard sense, $J_{u u}, J_{u v}$, and $K_{u v}$ must all be $>0$ and $J_{u u}>J_{u v}$. Therefore, $S$, $T, S^{\prime}$, and $T^{\prime}$ are all $>0$. The value of $U^{\prime}$ may be either positive or negative and, presumably, might accidently equal zero.

\section{THE MOLECULAR ORBITAL ENERGY OF THE CONFIGURATION $(e)^{2}$}

We now consider a molecular system in an electronic configuration that is entirely closed shell except for one open shell of the type $(e)^{2}$. Using the same orbitals for all states the energies from this configuration are

$$
\begin{aligned}
& { }^{1} E_{1}=\left\langle{ }^{1} \psi_{1}|H|{ }^{1} \psi_{1}\right\rangle=E_{0}+J_{u u}+K_{u v}, \\
& { }^{1} E_{2}=\left\langle{ }^{1} \psi_{2}|H|{ }^{1} \psi_{2}\right\rangle=E_{0}+J_{u u}-K_{u v}, \\
& { }^{1} E_{3}=\left\langle{ }^{1} \psi_{3}|H|{ }^{1} \psi_{3}\right\rangle=E_{0}+J_{u v}+K_{u v}, \\
& { }^{3} E=\left\langle{ }^{3} \psi|H|{ }^{3} \psi\right\rangle=E_{0}+J_{u v}-K_{u v},
\end{aligned}
$$

where $E_{0}$ arises from the closed shells and the one-electron part of the open shell. We still use $[u v]$ to represent the degenerate pair of orbitals.

We now see the impact that Eq. (7) has on these energies. If Eq. (7) holds for the integrals, i.e., if $k \neq 4$, then ${ }^{1} E_{2}={ }^{1} E_{3}$ as required by the more general theory. On the other hand, when $k=4$ and $U^{\prime} \neq 0$ and ${ }^{1} E_{2}$ and ${ }^{1} E_{3}$ are different.

It will be observed, of course, that Hund's rule is still obeyed for energies calculated at this level of approximation. This is because $K_{u v}$ must be positive and therefore ${ }^{1} E_{3}>{ }^{3} E$. We may also note that our analysis does not tell us that $K_{u v}$ $<J_{u v}$, although we expect it to be so normally.

\section{THE EFFECTS OF CONFIGURATION INTERACTION}

The discussion up to now tells us that, so far as energy effects present at the RHF level are concerned, Hund's rule should be obeyed for the type of configurations we are considering. The situation may change when configuration interaction (CI) is included in the wave functions, since excit- ed configurations might lower singlet energies more than triplet energies without violating any symmetry requirements. This is the point argued by Kollmar and Staemmler ${ }^{1}$ when they introduce their concept of dynamic spin polarization. We now examine in some detail the nature of the CI terms producing this effect.

Brillouin's theorem is invoked in discussions of the involvement of singly excited configurations in a $\mathrm{CI}$ wave function. The commonly made statement that Brillouin's theorem prevents single excitations from entering at the first order level is in general true, however, only for UHF wave functions. ${ }^{4}$ In particular, it does not apply (at least without modification) to open shell RHF wave functions of the Roothaan type. ${ }^{2}$ Nevertheless, the common form of Brillouin's theorem does apply to closed shell RHF functions because they represent a stationary point in the UHF energy functional but, of course, not necessarily its global minimum. ${ }^{4}$ At present, we are concerned with open shell RHF functions, and we investigate just what restrictions Brillouin-like theorems make on the first order interaction of configurations involving single excitations from open shell wave functions.

We consider a Roothaan type wave function with a closed shell configuration except for the $(e)^{2}$ part. The lowest energy singlet function (at the RHF level) is

$$
{ }^{1} \psi=\{|c \bar{c} a \bar{a} u \bar{v}|-|c \bar{c} a \bar{a} \bar{u} v|\} / \sqrt{2},
$$

where $c$ symbolizes paired orbitals in general, $a$ is one of the paired orbitals we single out for attention, and $[u v]$ is the degenerate pair as above. If in ${ }^{1} \psi$ we make the substitution, $u \rightarrow u+\lambda u^{\prime}$, where $u^{\prime}$ is orthogonal to all the occupied orbitals in ${ }^{1} \psi$, the Rayleigh quotient for the modified function has the form

$$
\left\langle{ }^{1} \psi^{\prime}|H|{ }^{1} \psi^{\prime}\right\rangle /\left\langle{ }^{1} \psi^{\prime} \mid{ }^{1} \psi^{\prime}\right\rangle=\frac{E_{\mathrm{HF}}+\lambda P+\lambda^{2} Q}{1+\lambda^{2}},
$$

where $E_{0}$ is the RHF energy of Eq. (9) and $P$ $=\left\langle{ }^{1} \psi|H|{ }^{1} \psi\left(u \rightarrow u^{\prime}\right)\right\rangle$. If $a, c$, and $[u v]$ are the optimum Roothaan HF orbitals for Eq. (9), then the standard argument tells us that Eq. (10) must take on its minimum at $\lambda=0$, a possibility only if $P=0$. Single excitations of the type $u \rightarrow u^{\prime}$ or $v \rightarrow v^{\prime}$ therefore do not enter into $\mathrm{CI}$ wave functions to first order.

The argument develops differently when we consider substitutions of the type $a \rightarrow a+\lambda a^{\prime}$, where, again, $a^{\prime}$ must be orthogonal to all of the occupied orbitals of ${ }^{1} \psi$. In this case the Rayleigh quotient has the form

$$
\begin{aligned}
& \left\langle{ }^{1} \psi^{\prime}|H|{ }^{1} \psi^{\prime}\right\rangle /\left\langle{ }^{1} \psi^{\prime} \mid{ }^{1} \psi^{\prime}\right\rangle \\
& =\frac{E_{\mathrm{HF}}+\lambda P+\lambda^{2} Q+\lambda^{3} R+\lambda^{4} S}{1+2 \lambda^{2}+\lambda^{4}} .
\end{aligned}
$$

As before, one obtains $P=0$, but it now has the more complicated form

$$
\begin{aligned}
\left\langle{ }^{1} \psi|H|^{1} \psi(a \rightarrow a)\right\rangle=0, & \\
{ }^{1} \psi\left(a \rightarrow a^{\prime}\right)= & \left\{\left|c \bar{c} a \bar{a}^{\prime} u \bar{v}\right|-\left|c \bar{c} a \bar{a}^{\prime} \bar{u} v\right|\right\} / 2 \\
& -\left\{\left|c \bar{c} \bar{a} a^{\prime} u \bar{v}\right|-\left|c \bar{c} \bar{a} a^{\prime} \bar{u} v\right|\right\} / 2 .
\end{aligned}
$$

Therefore, there is a single excitation singlet function that does not enter into a CI in first order. The new situation, 

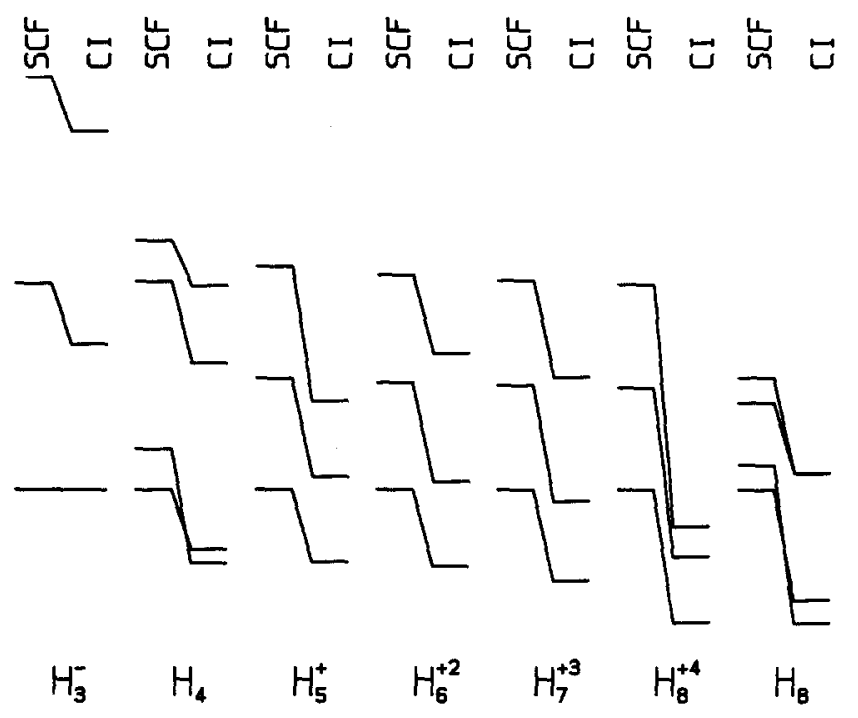

FIG. 1. Comparison of SCF and CI energies for the four electron systems $\mathrm{H}_{3}^{-}$to $\mathrm{H}_{8}^{+4}$ and the eight electron system $\mathrm{H}_{8}$. The SCF energies for all of the lowest ${ }^{3} A_{2}$ SCF energies are placed on one horizontal line so that the remainder of the values are easily intercompared.

however, is that Eq. (12) has two open shells and there is another independent singlet function ${ }^{5}$ that arises from this single excitation. In general, when there is more than one spin coupling, there is no unique way of writing eigenfunctions of the total spin. In the present situation, however, Brillouin's theroem singles out one particular spin coupling, and if the other is taken orthogonal to it, it is unique, also.

$$
\begin{aligned}
{ }^{1} \psi\left(a \rightarrow a^{\prime}\right)^{\prime}= & \sqrt{2}\left\{\left|c \bar{c} a a^{\prime} \bar{u} \bar{v}\right|+\left|c \bar{c} \bar{a} \bar{a}^{\prime} u v\right|\right\} / \sqrt{3} \\
& -\left\{\left|c \bar{c} a \bar{a}^{\prime} u \bar{v}\right|+\left|c \bar{c} a \bar{a}^{\prime} \bar{u} v\right|\right\} / \sqrt{1} \overline{2} \\
& -\left\{\left|c \bar{c} \bar{a} a^{\prime} u \bar{v}\right|+\left|c \bar{c} \bar{a} a^{\prime} \bar{u} v\right|\right\} / \sqrt{ } \overline{1} \overline{2}
\end{aligned}
$$

The matrix element of the Hamiltonian between Eq. $\left(12^{\prime}\right)$ and the RHF function is seen to be

$$
\left\langle{ }^{1} \psi|H|^{1} \psi\left(a \rightarrow a^{\prime}\right)^{\prime}\right\rangle=\left[a^{\prime} u \mid a u\right]-\left[a^{\prime} v \mid a v\right],
$$

and the variation theorem does not require this to be zero. Symmetry might still influence the matrix element in Eq. (13), but it will not be zero if $u^{2}-v^{2}$ and $a a^{\prime}$ possess a nonzero intersection in their species sets. Thus, the second singlet spin coupling can enter the CI wave function in first order and may have a correspondingly large effect on the CI energy in second order.

When the same analysis is carried out for the lowest triplet state, we find that single excitations from the open shell and one of the possible spin couplings from single excitations of a closed shell are functions that do not enter the CI in first order. With triplet functions, however, there are three possible spin couplings in a configuration having two open shells, and two first order terms in the CI functions are not prohibited by the variation principle:

$$
\begin{aligned}
& \left\langle{ }^{3} \psi|H|^{3} \psi\left(a \rightarrow a^{\prime}\right)^{\prime}\right\rangle=\sqrt{3}\left\{\left[a^{\prime} u \mid a u\right]-\left[a^{\prime} v \mid a v\right]\right\} / 2, \\
& \left\langle{ }^{3} \psi|H|^{3} \psi\left(a \rightarrow a^{\prime}\right)^{\prime \prime}\right\rangle=\sqrt{3 / 8}\left\{\left[a^{\prime} u \mid a u\right]+\left[a^{\prime} v \mid a v\right]\right\}
\end{aligned}
$$

where for cases of interest to us the matrix element in Eq. (14b) is normally zero because of spatial symmetry.

The effect of these matrix elements on the energy can be approximated to second order as

$$
E^{(2)}=-\left|\left\langle 0|H| a \rightarrow a^{\prime}\right\rangle\right|^{2} /\left(E_{e}-E_{0}\right) .
$$

Comparing Eq. (14a) with Eq. (13) one sees that for each of the possible $a \rightarrow a^{\prime}$ excitations the lowering in energy for the triple case is only $3 / 4$ that of the singlet if the corresponding excitation energies are approximately the same. A considerable difference in the excitation energies could reduce the difference in singlet and triplet CI stabilizations, but this was not observed in these calculations.

A criterion that Hund's rule be violated may now be stated. If

$$
\sum_{a, a^{\prime}}(1 / 4)\left\{\left[a u \mid a^{\prime} u\right]-\left[a v \mid a^{\prime} v\right]\right\}^{2} /\left(E_{e}-E_{0}\right)>2 K_{u v},
$$

we expect the lowest energy state to be singlet.

\section{THE INFLUENCE OF SYMMETRY ON THE SIZE OF $\boldsymbol{K}_{u v}$}

The inequality in Eq. (16) is not likely to be satisfied unless $K_{u v}$ is small. We may now show how this is likely to be the case only when $[u v]$ is a basis for the representation in Eq. (1) with $k=4$.

When one uses the LCAO method for constructing molecular wave functions, the AOs used can be sorted into symmetrically equivalent sets. Symmetry orbitals are then constructed by subjecting one AO to projection operators from the group

$$
\sigma=(f / g) \sum_{p} D\left(R_{p}\right)_{11} R_{p} X .
$$

When $k=4$ the representation matrices have one of the forms

$$
\left[\begin{array}{rr} 
\pm 1 & 0 \\
0 & \pm 1
\end{array}\right] \text { or }\left[\begin{array}{rr}
0 & \pm 1 \\
\pm 1 & 0
\end{array}\right]
$$

where all possible combinations of the signs occur. For the groups we are considering and $k=4$, Eq. (17) frequently has the effect of dividing the AOs into two distinct, nonadjacent sets. Put another way, $u$ and $v$ are nonbonding orbitals that are linear combinations of disjoint sets of AOs; the product $u v$ does not contain in its sum the square of any of the AOs.

For the moment let us consider a ring of equivalent AOs. If the ring has fourfold symmetry and the representation involved has $k=4$, the product $u v$ will decrease exponentially as the ring size increases, and consequently $K_{u v}$ also will decrease exponentially.

This behavior must be contrasted to what happens if $k \neq 4$. The product function $u v$ then has terms in it that are the square of AOs. These do not go to zero as the size of the ring increases. Consequently, $K_{u v}$ does not go to zero either.

When $k \neq 4, J_{u u}, J_{u v}$, and $K_{u v}$ must be related by Eq. (7). Since $J_{u u}$ and $J_{u v}$ are never zero as the ring increases in size, $K_{u v}$ cannot approach zero since $J_{u u}$ and $J_{u v}$ cannot be equal if $u \neq v$. Therefore, only when $k=4$, Eq. (7) does not apply, and the value of $K_{u v}$ is unconnected to the $J$ integrals, do we expect the exchange integral to be quite small for AOs 
TABLE I. Character table for $\mathrm{D}_{7}$.

\begin{tabular}{llllllll}
\hline & $E$ & $2 C_{7}$ & $2 C_{7}^{3}$ & $2 C_{7}^{3}$ & $7 C_{2}^{\prime}$ & \multicolumn{1}{c}{$\epsilon=2 \pi / 7$} \\
\hline$A_{1}$ & 1 & 1 & 1 & 1 & 1 & $x^{2}+y^{2}, z^{2}$ \\
$A_{2}$ & 1 & 1 & 1 & 1 & -1 & $z$ & \\
$E_{1}$ & 2 & $2 \cos \epsilon$ & $2 \cos 2 \epsilon$ & $2 \cos 3 \epsilon$ & 0 & $(x, y)$ & $(x z, y z)$ \\
$E_{2}$ & 2 & $2 \cos 2 \epsilon$ & $2 \cos 4 \epsilon$ & $2 \cos 6 \epsilon$ & 0 & & $\left(x^{2}-y^{2}, x y\right)$ \\
$E_{3}$ & 2 & $2 \cos 3 \epsilon$ & $2 \cos 6 \epsilon$ & $2 \cos 9 \epsilon$ & 0 & \\
\hline
\end{tabular}

in a ring. If the ring is expanded enough $K_{u v}$ could be very small.

This may be contrasted with the behavior of the left-

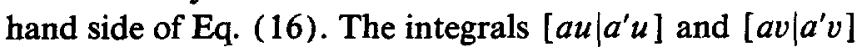
do not involve orbitals that are linear combinations of AOs sorted into nonadjacent sets, and the left-hand side of $\mathrm{Eq}$. (16) should be much less sensitive to changes in interatomic distances than the right. Therefore, situations should arise in which Eq. (16) is true, and Hund's rule is violated.

\section{MODEL CALCULATIONS WITH RINGS OF H ATOMS}

A series of model calculations have been made that illustrate the theory we have outlined. These were performed at the SCF and $\mathrm{CI}$ levels for rings of $\mathrm{H}$ atoms and may be described as $\mathrm{H}_{3}^{-}, \mathrm{H}_{4}, \mathrm{H}_{5}^{+}, \mathrm{H}_{6}^{+2}, \mathrm{H}_{7}^{+3}, \mathrm{H}_{8}^{+4}$, and $\mathrm{H}_{8}$. An octahedron of $\mathrm{H}_{6}^{+2}$ and a square plane of $\mathrm{H}_{9}^{-}$are also considered. The $\mathrm{H}$ atom systems were all calculated at the minimal level with the Huzinaga 4 Gaussian orbital ${ }^{6}$ at each proton and, with only one exception, an adjacent distance of $1.08 \AA$ in all of the rings and the octahedron.

Figure 1 shows the energy levels calculated at the SCF and $\mathrm{CI}$ level for the $\mathrm{H}_{n}$ ring systems. The pattern of violation of Hund's rule is exactly as described in the theory and occurs only for $\mathbf{H}_{4}$ and $\mathrm{H}_{8}$, the instances for which $k=4$ among the two-dimensional representations involved. In particular, we note that, in spite of the fourfold symmetry in the system, $\mathrm{H}_{8}^{+4}$ does not violate Hund's rule since the $E_{1}$ representation matrices of the group all have $k=8$. Character tables for $D_{7}$ and $D_{8}$ are not given in standard reference works; for convenience we give them in Tables I and II.

The various values of $J_{u u}, J_{u v}$, and $K_{u v}$ are plotted in Fig. 2 as a function of the ring size. In all cases, except when $k=4$, these quantities show consistent trends in their values as the ring changes size. In the two exceptional cases $K_{u v}$ is abnormally small. The values of the decrease in energy from the $\mathrm{CI}$ can be seen in Fig. 1. These all change consistently with ring size and show no anomalies for the $k=4$ cases.

TABLE II. Character table for $\mathrm{D}_{\mathbf{8}}$.

\begin{tabular}{|c|c|c|c|c|c|c|c|c|c|}
\hline & $E$ & $2 \mathrm{C}_{8}$ & $2 C_{4}$ & $2 C_{8}^{3}$ & $C_{2}$ & $4 C_{2}^{\prime}$ & $4 C_{2}^{\prime \prime}$ & & \\
\hline$A_{1}$ & 1 & 1 & 1 & 1 & 1 & 1 & 1 & & $x^{2}+y^{2}, z^{2}$ \\
\hline$A_{2}$ & 1 & 1 & 1 & 1 & 1 & -1 & -1 & $z$ & \\
\hline$B_{1}$ & 1 & -1 & 1 & -1 & 1 & 1 & -1 & & \\
\hline$B_{2}$ & 1 & -1 & 1 & -1 & 1 & -1 & 1 & & \\
\hline$E_{1}$ & 2 & $\sqrt{ } 2$ & 0 & $-\sqrt{ } 2$ & -2 & 0 & 0 & $(x, y)$ & $(x z, y z)$ \\
\hline$E_{2}$ & 2 & 0 & -2 & 0 & 2 & 0 & 0 & & $\left(x y, x^{2}-y^{2}\right)$ \\
\hline$E_{3}$ & 2 & $-\sqrt{ } 2$ & 0 & $\sqrt{2}$ & -2 & 0 & 0 & & \\
\hline
\end{tabular}

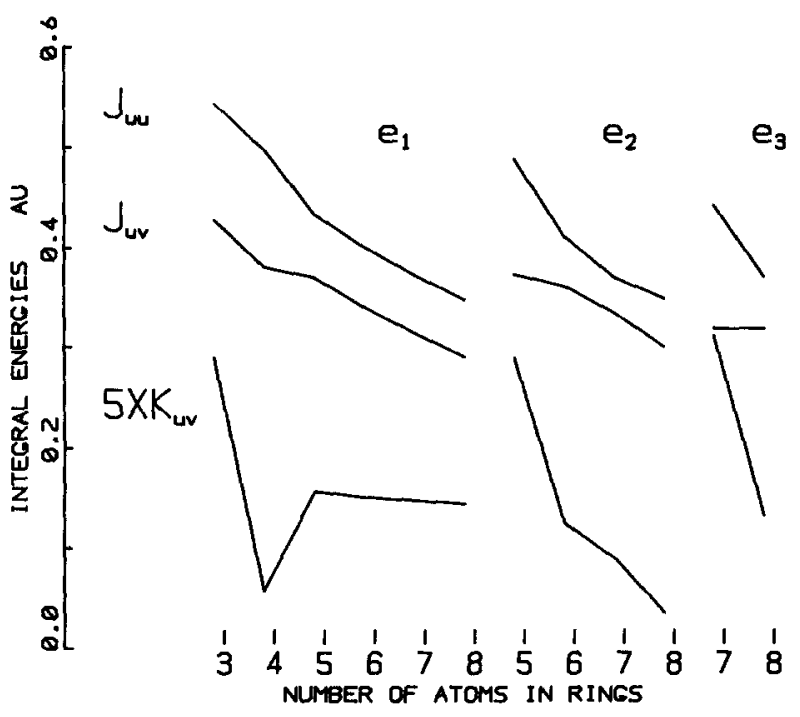

FIG. 2. The two electron integrals $J_{u v}, J_{u v}$, and $K_{u v}$ for the degenerate orbital pairs in the $H_{n}$ ring systems. As $n$ increases a larger number of different two-dimensional irreducible representations appear in the group.

For these $\mathbf{H}$ ring systems the conclusion is clear: the violation of Hund's rule when $k=4$ is the result of abnormally small values of the exchange integral $K_{u s}$ and not of abnormally high CI stabilizations.

\section{OTHER SYSTEMS WITH FOURFOLD SYMMETRY}

Calculations on two other model systems involving all $\mathrm{H}$ atoms have been carried out: (a) $\mathrm{H}_{6}^{+2}$ arranged in a regular octahedron and (b) $\mathrm{H}_{9}^{-}$arranged in a square. For these we have retained the same form of the $\mathrm{H} \mathrm{AO}$ as was used in the ring systems. The octahedral model system was calculated for two different internuclear distances to demonstrate that the breakdown of Hund's rule can depend on system size. The results on $\mathrm{H}_{9}^{-}$suggest that Hund's rule will always be satisfied, and this system was treated at the $1.08 \AA$ nearest neighbor distance, only.

(a) An octahedron has several fourfold axes of rotation as well as threefold and also $S_{6}$ operations. We may make the same type of analysis as we have made for the ring compounds. The situation here is somewhat more complicated since the open shell is threefold rather than twofold degenerate. If we designate the orbitals $[u v w]$, there are three independent two electron integrals $J_{u u}, J_{u v}$, and $K_{u v}$ with no relation like Eq. (7) among them. ${ }^{7}$ The triply degenerate orbitals for this system can have the same type of nonbonding character as the doubly degenerate orbitals in the fourfold rings have. We therefore expect the same type of behavior with internuclear separation. Figure 3 shows the energy levels in this case for nearest neighbor distances of 1.08 (the same as the ring calculations) and $1.5 \AA$. Whether Hund's rule breaks down depends upon the distance between the atoms. The exchange integral for the nonbonding orbitals decreases with distance so much faster than the Coulomb integrals that the singlet state is eventually lower than the triplet state as is true for the fourfold ring systems.

Unfortunately, octahedral symmetry, related to one of the Euclidean solids, is not part of any systematic sequence 


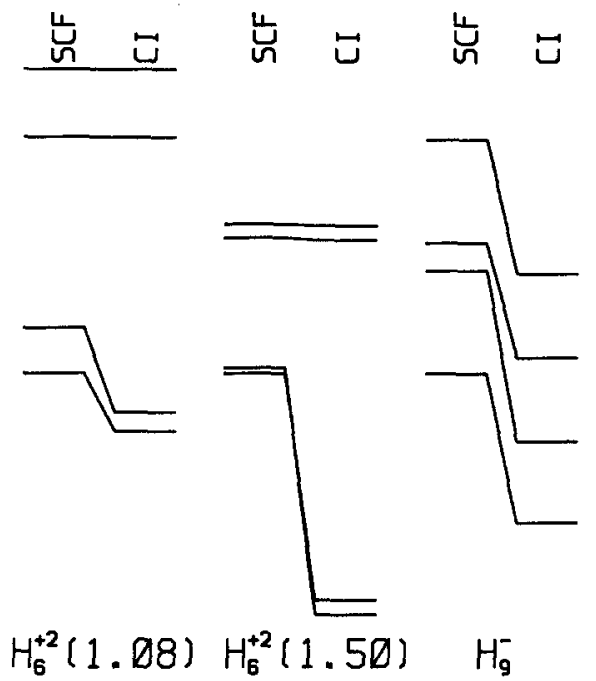

FIG. 3. Comparison of SCF and CI energies for two octahedral arrangements of $\mathrm{H}_{6}^{+2}$ and square planar $\mathrm{H}_{9}^{-}$. The lowest triplet SCF energy was placed on a common line to facilitate intercomparison.

of group symmetries. It is therefore unlikely that we will ever be able to investigate any trends containing the present example.

While discussing this system we mention that octahedral $\mathrm{H}_{6}^{+}$having an $(f)^{3}$ configuration does satisfy Hund's multiplicity rule. The ${ }^{4} A_{1 g}$ is the lowest energy for this model system at both of the distances calculated here.

(b) Figure 3 also shows the energies for the lowest configuration of $\mathrm{H}_{9}^{-}$, which has an $(e)^{2}$ open shell; Hund's rule is satisfied for this system. Only one distance has been calculated, but one does not expect the type of behavior found with the octahedron. The argument is as follows: $\mathrm{H}_{9}^{-}$is again a case where Eq. (7) does not apply, but, unlike the ring systems,

$$
K_{u v}>\left(J_{u u}-J_{u v}\right) / 2
$$

rather than being quite small. The result is a level reversal compared to the ring systems. The exchange integral is large in this case because the [uv] orbitals cannot have a purely nonbonding form. If we label the atoms in the square as

$\begin{array}{lll}1 & 2 & 3 \\ 4 & 5 & 6 \\ 7 & 8 & 9\end{array}$

one sees that atom groups $\{1,3,7,9\}$ and $\{2,4,6,8\}$ are disjoint symmetrically equivalent sets. Linear combinations of these that form $e$-type functions necessarily have both bonding and nonbonding character within the same orbital. The corresponding exchange integral is not small and would not approach zero if the distances were increased without bound.

$\mathrm{H}_{9}^{-}$is striking in the large amount of electron correlation represented by double and higher excitations in the $\mathrm{CI}$ wave function. The involvement is so great that an MO picture is not likely to be useful in this system. We pursue this point further in a later work.

\section{CYCLOBUTADIENE}

Cyclobutadiene has generated interest as a simple case where Hückel theory predicts an unstable system. It is now known that cyclobutadiene is most stable in a singlet state with rectangular geometry. ${ }^{8}$ Nevertheless, the electronic states of the $\pi$ electrons when the molecule is constrained to be square are of theoretical importance. This is, of course, a system that also shows the violation of Hund's rule as we have discussed. Voter and Goddard have discussed the MOCI results of cyclobutadiene $e^{9}$ and have pointed out the small size of the exchange integral $K_{u v}$ for the $\pi$ orbitals of this molecule.

\section{DISCUSSION}

The dynamic spin polarization defined by Kollmar and Staemmler is seen to be a concept arising from the deficiencies in the open-shell RHF-MOCI zeroth order wave function and is expected to be present for all wave functions of this type. It is not an effect of electron correlation but is a result of the spin degeneracy ${ }^{10}$ in systems with two or more open shells.

Simple Hückel theory as applied to ring systems is frequently credited with yielding a clearcut picture of the states in these systems. We now see, however, that those cases where the ring has fourfold symmetry require more delicate interactions of different spin states and a knowledge of the relative sizes two electron integrals to arrive at a qualitatively correct picture of the states.

The influence of fourfold symmetry we have been discussing appears to arise from quite deep topological considerations connected with the fourfold nature of our threedimensional space. At present, we have probably only uncovered the effect and have not succeeded in arriving at anything like a complete description of its ontology.

\section{SUMMARY}

We have ranged over a number of topics dealing with symmetry and molecular energies. We have shown that systems with fourfold symmetry are likely candidates for the breakdown of Hund's multiplicity rule. Examples have been discovered that do satisfy the rule, however. Brillouin-like theorems permit some first order corrections to open shell RSCF wave functions, and the energy lowering due to this helps the breakdown. Nevertheless, the crucial condition in the systems treated has been the small size of an exchange integral, $K_{u v}$, for some of them. Under these circumstances, the values of the energies established at the level of the independent particle approximation can be interchanged by single excitation interactions in the $\mathrm{CI}$ and are, hence, qualitatively changed.

\footnotetext{
${ }^{1}$ H. Kollmar and V. Staemmler, Theor. Chim. Acta 48, 223 (1978).

${ }^{2}$ C. C. J. Roothaan, Rev. Mod. Phys. 32, 1174 (1960).

${ }^{3}$ W. J. Hehre and J. A. Pople, J. Am. Chem. Soc. (in press).

${ }^{4}$ See, for example, The Variation Method in Quantum Chemistry, edited by

S. T. Epstein (Academic, New York, 1974), Chap. III.

${ }^{5}$ For a very complete treatment see Spin Eigenfunctions, edited by $\mathbf{R}$.
} 
Pauncz (Plenum, New York, 1979).

${ }^{6}$ S. Huzinaga, J. Chem. Phys. 42, 1293 (1965).

${ }^{7}$ The situation for the octahedron should be contrasted with that in an atom. One of the triply degenerate species in $O_{h}$ behaves just like the coordinate variables $x, y$, and $z$, and also like $p_{x}, p_{y}$, and $p_{z}$ in an atom. Nevertheless, in the spherical symmetry of the atom, there are relations between two electron integrals, one example being $J_{x x}-J_{x y}=2 K_{x p}$. The lower symmetry of the octahedron does not support such relations.

${ }^{8}$ S. Masamune, F. A. Souto-Bachiller, T. Machiguchi, and J. E. Bertie, J. Am. Chem. Soc. 100, 4889 (1978); D. W. Whitman and B. K. Carpenter, ibid. 102, 4272 (1980); 104, 6473 (1982).

${ }^{9}$ A. F. Voter and W. A. Goddard, J. Am. Chem. Soc. 108, 2830 (1986).

${ }^{10}$ P.-O. Löwdin, Coll. Int. Centre Nat. Rech. Sci. 82, 23 (1958); see also Ref. 5. 